\title{
The Stability of High Order Max-Type Difference Equation
}

\author{
Han Cai-hong", Li Lue, Tan Xue \\ School of Mathematics and Statistics, Guangxi Normal University, Guilin, China
}

Email address:

hchong_math@163.com (Han Cai-hong)

${ }^{*}$ Corresponding author

\section{To cite this article:}

Han Cai-hong, Li Lue, Tan Xue. The Stability of High Order Max-Type Difference Equation. Applied and Computational Mathematics. Vol. 5, No. 2, 2016, pp. 51-55. doi: 10.11648/j.acm.20160502.13

Received: February 5, 2016; Accepted: March 28, 2016; Published: April 7, 2016

Abstract: In this paper, we investigate the stability of following max-type difference equation $x_{n+1}=p+\sum_{i=1}^{t} a_{i} x_{n-m_{i}} \max \left\{\frac{b_{1}}{x_{n-n_{1}}}, \cdots, \frac{b_{r}}{x_{n-n_{r}}}\right\} \quad, \quad$ where $\quad 0 \leq m_{1}<m_{2}<\cdots<m_{t}, 0 \leq n_{1}<n_{2}<\cdots<n_{r} \quad, \quad$ with $\left\{m_{1}, m_{2}, \cdots, m_{t}\right\} \cap\left\{n_{1}, n_{2}, \cdots, n_{r}\right\}=\phi, \quad a_{i}>0 \quad(i=1,2, \cdots t), \quad b_{j}>0 \quad(j=1,2, \cdots r) \quad$ and $\quad p>\max \left\{b_{1}, \cdots, b_{r}\right\} \cdot \sum_{i=1}^{t} a_{i}$, the initial values are positive. By constructing a system of equations and binary function, we show the equation has a unique positive equilibrium solution, and the positive equilibrium solution is globally asymptotically stable. The conclusion of this paper extends and supplements the existing results.

Keywords: Difference Equations, Positive Solution, Convergence, Globally Stable

\section{Introduction}

In mathematics, recursive relation, which is difference equation, is a kind of recursion formula to define a sequence: the sequence of each item is defined as a function. Difference equation is the discretization of differential equations. The difference system is described the mathematical model of discrete system, it is an important branch of dynamical system, the application of its theory is rapidly broadening to various fields, such as economics, ecology, physics, engineering, control theory, computer science and so on (see [1-4]). The stability and global behavior is one of the hot spots in researches about difference equation model, the conclusion has a certain guiding role to production practices.

In recent years, more and more researches on the dynamic behaviors of higher order nonlinear difference equations have been studied (see [5-19]). One of the classes of such difference equations are max-type difference equations (see [10-19]).

In [16], Amleh studied the nonlinear difference equation $x_{n+1}=p+\frac{x_{n-1}}{x_{n}}$, showed that the unique positive equilibrium solution $\bar{x}=p+1$ is globally asymptotically stable:

In [17], Fan studied the higher order difference equation $x_{n+1}=f\left(x_{n}, x_{n-k}\right)$, and gave a sufficient condition for its global asymptotical stability, these results are applied to the

difference equation $x_{n+1}=a+\frac{x_{n-k}}{x_{n}}$.

In [18], Sun studied global behavior of the max-type difference equation $x_{n+1}=\max \left\{1 / x_{n-m}, A_{n} / x_{n-r}\right\}$, proved that if $A_{n} \in(0,1)$ and $\sup A_{n}<1$ is a periodic sequence, then every positive solution of this equation is eventually periodic with period $2 \mathrm{~m}$.

In [19], Stević studied behavior of positive solutions of the following max-type system of difference equations,

$$
x_{n+1}=\max \left\{c, \frac{y_{n}^{p}}{x_{n-1}^{p}}\right\}, y_{n+1}=\max \left\{c, \frac{x_{n}^{p}}{y_{n-1}^{p}}\right\}
$$

proved that if $p, c \in(0,1)$ then every positive solution of the 
system converges to $(1,1)$.

In this paper, we investigate the global stability of following max-type difference equation

$$
x_{n+1}=p+\sum_{i=1}^{t} a_{i} x_{n-m_{i}} \max \left\{\frac{b_{1}}{x_{n-n_{1}}}, \cdots, \frac{b_{r}}{x_{n-n_{r}}}\right\}
$$

where $n=0,1,2, \cdots, \quad 0 \leq m_{1}<m_{2}<\cdots<m_{t}, 0 \leq n_{1}<n_{2}<$ $\cdots<n_{r} \quad$ with $\quad\left\{m_{1}, m_{2}, \cdots, m_{t}\right\} \cap\left\{n_{1}, n_{2}, \cdots, n_{r}\right\}=\varphi, \quad a_{i}>0$ $(i=1,2, \cdots t) \quad, \quad b_{j}>0(j=1,2, \cdots r) \quad$ and $\quad P>\max \left\{b_{1}\right.$, $\left.\cdots, b_{r}\right\} \cdot \sum_{i=1}^{t} a_{i}$, the initial values are positive. By constructing a system of equations and binary function, we will formulate and prove the equation has a unique positive equilibrium solution, and the positive equilibrium solution is globally asymptotically stable. The conclusion of this paper extends and supplements the existing results, this conclusion has a certain guiding role to production practices as a mathematical model.

For convenience, we denote $l=\max \left\{m_{t}, n_{r}\right\}, A=\sum_{i=1}^{t} a_{i}$, $B=\max \left\{b_{1}, \cdots, b_{r}\right\}$. So $p>A B$.

\section{Some Definitions}

In this section we will introduce some definitions (see [20]) which will be needed.

Definition A. [20] Let $I$ be some interval of numbers and let $f: I \times I \rightarrow I$ be a continuously difference function.

A difference equation of order $(k+1)$ is an equation of the form

$$
x_{n+1}=f\left(x_{n}, x_{n-1}, \cdots, x_{n-k}\right), n=0,1, \cdots
$$

A point $\bar{x} \in I$ is called equilibrium solution of the difference equation if $\bar{x}=f(\bar{x}, \bar{x}, \cdots, \bar{x})$, that is $x_{n}=\bar{x}$ for all $n \geq-k$.

Definition B. [20] The equilibrium $\bar{x}$ is called locally stable if for every $\varepsilon>0$, there exists $\delta>0$ such that if $\left\{x_{n}\right\}_{n=-k}^{\infty}$ is a solution of difference equation with initial values satisfied $\left|x_{-k}-\bar{x}\right|+\left|x_{1-k}-\bar{x}\right|+\cdots+\left|x_{0}-\bar{x}\right|<\delta$, then

$$
\left|x_{n}-\bar{x}\right|<\varepsilon \text { for all } n \geq-k .
$$

Definition C. [20] The equilibrium $\bar{x}$ is called a global attractor if for every solution $\left\{x_{n}\right\}_{n=-k}^{\infty}$ of difference equation, we have $\lim _{n \rightarrow \infty} x_{n}=\bar{x}$.

Definition D. [20] The equilibrium $\bar{x}$ of difference equation is called globally asymptotically stable if $\bar{x}$ is locally stable, and $\bar{x}$ is also a global attractor of the difference equation.

\section{Main Results}

In this section we formulate and prove some lemmas and main theorems in this paper, obtain that every positive solution of (1) has to be the ultimate form of globally asymptotically stable.

Theorem 1. Equation (1) has a unique positive equilibrium solution $\bar{x}=p+A B$.

Proof. Since

$$
\bar{x}=p+\sum_{i=1}^{t} a_{i} \bar{x} \max \left\{\frac{b_{1}}{\bar{x}}, \cdots, \frac{b_{r}}{\bar{x}}\right\}
$$

we have $\bar{x}=p+A \bar{x} \cdot \frac{B}{\bar{x}}=p+A B>p$, so equation (1) has a unique positive equilibrium $\bar{x}=p+A B$. \#

Equation $p+\sum_{i=1}^{t} a_{i} x \cdot \max \left\{\frac{b_{1}}{p}, \cdots, \frac{b_{r}}{p}\right\}=x$, that is $p+A x \cdot \frac{B}{p}=x$, the only fixed point for the solution of this equation is $x=\frac{p^{2}}{p-A B}$, denote by $D$, that is $D=\frac{p^{2}}{p-A B}$. Because

$$
D-p=\frac{p^{2}}{p-A B}-p=\frac{p^{2}-p(p-A B)}{p-A B}=\frac{p A B}{p-A B}>0
$$

so $D>p$.

Lemma 1. For any real number $H \geq D$, if initial values $x_{0}, x_{-1}, \cdots, x_{-l} \in[p, H]$, then $x_{n} \in[p, H](n \geq 1)$.

Proo $f$. Since $H \geq D=\frac{p^{2}}{p-A B}$, so $H \geq \frac{p^{2}+A B H}{p}$, for any $x_{0}, x_{-1}, \cdots, x_{-l} \in[p, H]$, we have

$$
p \leq x_{1}=p+\sum_{i=1}^{t} a_{i} x_{-m_{i}} \max \left\{\frac{b_{1}}{x_{-n_{1}}}, \cdots, \frac{b_{r}}{x_{-n_{r}}}\right\}
$$$$
\leq p+\frac{A B H}{p}=\frac{p^{2}+A B H}{p} \leq H,
$$$$
p \leq x_{2}=p+\sum_{i=1}^{t} a_{i} x_{1-m_{i}} \max \left\{\frac{b_{1}}{x_{1-n_{1}}}, \cdots, \frac{b_{r}}{x_{1-n_{r}}}\right\}
$$

$$
\leq p+\frac{A B H}{p}=\frac{p^{2}+A B H}{p} \leq H,
$$


suppose for every $n \leq k$, there is $x_{n} \in[p, H]$, then

$$
\begin{aligned}
p \leq x_{k+1} & =p+\sum_{i=1}^{t} a_{i} x_{k-m_{i}} \max \left\{\frac{b_{1}}{x_{k-n_{1}}}, \cdots, \frac{b_{r}}{x_{k-n_{r}}}\right\} \\
& \leq p+\frac{A B H}{p}=\frac{p^{2}+A B H}{p} \leq H .
\end{aligned}
$$

By induction, for every $n \geq 1$, we have $x_{n} \in[p, H]$. \#

Let $h_{0}=p, H_{0}=H \geq D$, for any $i \geq 0$, define the system of equations as follows:

$$
\begin{aligned}
& h_{i+1}=p+\sum_{i=1}^{t} a_{i} h_{i} \max \left\{\frac{b_{1}}{H_{i}}, \cdots, \frac{b_{r}}{H_{i}}\right\} \\
& H_{i+1}=p+\sum_{i=1}^{t} a_{i} H_{i} \max \left\{\frac{b_{1}}{h_{i}}, \cdots, \frac{b_{r}}{h_{i}}\right\}
\end{aligned}
$$

that is $h_{i+1}=p+A h_{i} \cdot \frac{B}{H_{i}}, H_{i+1}=p+A H_{i} \cdot \frac{B}{h_{i}}$.

Lemma 2. For every $n \geq 0$, there is

$$
h_{n} \leq h_{n+1}<\bar{x}<H_{n+1} \leq H_{n}
$$

and $\lim _{n \rightarrow \infty} H_{n}=\lim _{n \rightarrow \infty} h_{n}=\bar{x}$.

Proof. Obtained by above definition (2-3), we have

$$
\begin{gathered}
h_{0} \leq h_{1}=p+A h_{0} \cdot \frac{B}{H_{0}}=p+A p \cdot \frac{B}{H}<p+A B=\bar{x} \\
H_{0} \geq H_{1}=p+A H_{0} \cdot \frac{B}{h_{0}}=p+A H \cdot \frac{B}{p}>p+A B=\bar{x}
\end{gathered}
$$

so $p=h_{0} \leq h_{1}<\bar{x}<H_{1} \leq H_{0}=H$. Because

$$
\begin{aligned}
h_{1}=p+A h_{0} \cdot \frac{B}{H_{0}} \leq h_{2}= & p+A h_{1} \cdot \frac{B}{H_{1}} \\
& <p+A \bar{x} \cdot \frac{B}{\bar{x}}=p+A B=\bar{x}, \\
H_{1}=p+A H_{0} \cdot \frac{B}{h_{0}} \geq H_{2}= & p+A H_{1} \cdot \frac{B}{h_{1}} \\
& >p+A \bar{x} \cdot \frac{B}{\bar{x}}=p+A B=\bar{x},
\end{aligned}
$$

so $h_{0} \leq h_{1} \leq h_{2}<\bar{x}<H_{2} \leq H_{1} \leq H_{0}$.

By induction, there is $h_{n} \leq h_{n+1}<\bar{x}<H_{n+1} \leq H_{n}$ for every $n \geq 0$. That is

$$
\begin{aligned}
p=h_{0} \leq h_{1} \leq h_{2} \leq \cdots \leq h_{n} \leq \cdots<\bar{x}< \\
\cdots<H_{n} \leq \cdots \leq H_{2} \leq H_{1} \leq H_{0}=H .
\end{aligned}
$$

According to the monotone bounded theorem, we know the limits of $\left\{h_{n}\right\},\left\{\mathrm{H}_{n}\right\}$ are existence. Let $\bar{h}=\lim _{n \rightarrow \infty} h_{n}$, $\bar{H}=\lim _{n \rightarrow \infty} H_{n}$. Take limits on both sides of (2-3), then

$$
\begin{aligned}
& \bar{h}=p+\sum_{i=1}^{t} a_{i} \bar{h} \cdot \max \left\{\frac{b_{1}}{\bar{H}}, \cdots, \frac{b_{r}}{\bar{H}}\right\} \\
& \bar{H}=p+\sum_{i=1}^{t} a_{i} \bar{H} \cdot \max \left\{\frac{b_{1}}{\bar{h}}, \cdots, \frac{b_{r}}{\bar{h}}\right\}
\end{aligned}
$$

that is $\bar{h}=p+A \bar{h} \cdot \frac{B}{\bar{H}}, \quad \bar{H}=p+A \bar{H} \cdot \frac{B}{\bar{h}}$, therefore $\bar{h} \bar{H}=P \bar{H}+A B \bar{h}=P \bar{h}+A B \bar{H} \quad, \quad$ so $\quad(p-A B)$ $(\bar{h}-\bar{H})=0$. Since $p>A B$, so $\bar{h}=\bar{H}=p+A B$, that is $\lim _{n \rightarrow \infty} H_{n}=\lim _{n \rightarrow \infty} h_{n}=\bar{x} . \#$

Theorem 2. The unique equilibrium $\bar{x}=p+A B$ of equation (1) is locally stable.

Proof. Set $H=D=\frac{p^{2}}{p-A B}, h_{n}$ and $H_{n}$ as defined in Lemma 2. For every $\varepsilon>0$ with $0<\varepsilon<\min \{D-\bar{x}, \bar{x}-p\}$, according to Lemma 2 and local boundedness, there exists $n \geq 0$ such that $\bar{x}-\varepsilon<h_{n}<\bar{x}<H_{n}<\bar{x}+\varepsilon$.

Take $0<\delta=\min \left\{\bar{x}-h_{n}, H_{n}-\bar{x}\right\}$, that is $(\bar{x}-\delta, \bar{x}+\delta)$ $\subset\left[h_{n}, H_{n}\right]$. Then for every $x_{0}, x_{-1}, \cdots, x_{-l} \in(\bar{x}-\delta, \bar{x}+\delta)$, we have

$$
\begin{gathered}
x_{1}=p+\sum_{i=1}^{t} a_{i} x_{-m_{i}} \max \left\{\frac{b_{1}}{x_{-n_{1}}}, \cdots, \frac{b_{r}}{x_{-n_{r}}}\right\} \\
\leq p+\sum_{i=1}^{t} a_{i} H_{n} \max \left\{\frac{b_{1}}{h_{n}}, \cdots, \frac{b_{r}}{h_{n}}\right\}=H_{n+1} \leq H_{n}, \\
x_{1} \geq p+\sum_{i=1}^{t} a_{i} h_{n} \max \left\{\frac{b_{1}}{H_{n}}, \cdots, \frac{b_{r}}{H_{n}}\right\}=h_{n+1} \geq h_{n},
\end{gathered}
$$

that is $x_{1} \in\left[h_{n}, H_{n}\right] \subset(\bar{x}-\varepsilon, \bar{x}+\varepsilon)$.

Similarly, by induction, there is $x_{n} \in\left[h_{n}, H_{n}\right] \subset$ $(\bar{x}-\varepsilon, \bar{x}+\varepsilon)$ for every $n \geq-l$. According to theorem $\mathrm{B}$, the equilibrium $\bar{x}=p+A B$ is locally stable. \#

Theorem 3. The unique equilibrium solution $\bar{x}=p+A B$ 
of equation (1) is globally asymptotically stable.

Proof. In Theorem 2, we have proved $\bar{x}=p+A B$ is locally stable, then we will prove $\bar{x}=p+A B$ is global attractor.

Set $H=\max \left\{x_{1}, \cdots, x_{l+1}, D\right\}, h_{n}$ and $H_{n}$ as defined in Lemma 2. Following Lemma 1 , for every $n \geq 1$, there is $x_{n} \in\left[h_{0}, H_{0}\right]=[p, H]$. So

$$
\begin{gathered}
h_{1}=p+A h_{0} \cdot \frac{B}{H_{0}} \leq x_{(l+1)+1} \\
=p+\sum_{i=1}^{t} a_{i} x_{l+1-m_{i}} \max \left\{\frac{b_{1}}{x_{l+1-n_{1}}}, \cdots, \frac{b_{r}}{x_{l+1-n_{r}}}\right\} \\
\leq p+A H_{0} \cdot \frac{B}{h_{0}}=H_{1}, \\
h_{1}=p+A h_{0} \cdot \frac{B}{H_{0}} \leq x_{(l+2)+1} \\
=p+\sum_{i=1}^{t} a_{i} x_{l+2-m_{i}} \max \left\{\frac{b_{1}}{x_{l+2-n_{1}}}, \cdots, \frac{b_{r}}{x_{l+2-n_{r}}}\right\} \\
\leq p+A H_{0} \cdot \frac{B}{h_{0}}=H_{1} .
\end{gathered}
$$

By induction, there is $x_{n} \in\left[h_{1}, H_{1}\right]$ for every $n \geq(l+1)+1$.

Similarly, we have $x_{n} \in\left[h_{1}, H_{1}\right]$ for every $n \geq 2(l+1)+1$. By induction, $x_{n} \in\left[h_{k}, H_{k}\right]$ for every $n \geq k(l+1)+1$ where $k=0,1, \cdots$.

Following Lemma 2, we know $\lim _{n \rightarrow \infty} H_{n}=\lim _{n \rightarrow \infty} h_{n}=\bar{x}$, so $\lim _{n \rightarrow \infty} x_{n}=\bar{x}$. By Definition C, we know $\bar{x}=p+A B$ is global attractor.

According to Definition D, it is obviously that the equilibrium $\bar{x}=p+A B$ of equation (1) is globally asymptotically stable. \#

\section{Example}

Consider one of example of differential equation (1):

$$
x_{n+1}=20+\left(2 x_{n-2}+0.5 x_{n-4}+3.5 x_{n-5}\right) \cdot \max \left\{\frac{0.5}{x_{n-3}}, \frac{3}{x_{n-6}}, \frac{1.5}{x_{n-9}}\right\} \quad n=0,1, \cdots
$$

where the initial values $x_{0}, x_{-1}, \cdots, x_{-9} \in(0,+\infty)$. Obviously, it satisfies the conditions of Theorem 3 , so the unique equilibrium $\quad \bar{x}=38$ of equation (4) is globally asymptotically stable. By giving the initial value assignment, the following figures 1-2 show the global asymptotic stability.

If initial values $x_{0}=x_{-1}=\cdots=x_{-9}=10$, equilibrium $\bar{x}=38$ is globally asymptotically stable (see Figure 1).

If initialvalues $x_{-3}=x_{-6}=x_{-9}=0.05, x_{0}=x_{-1}=x_{-2}=x_{4}=x_{-5}=$ $x_{-7}=x_{-8}=10$, equilibrium $\bar{x}=38$ is globally asymptotically (see Figure 2).

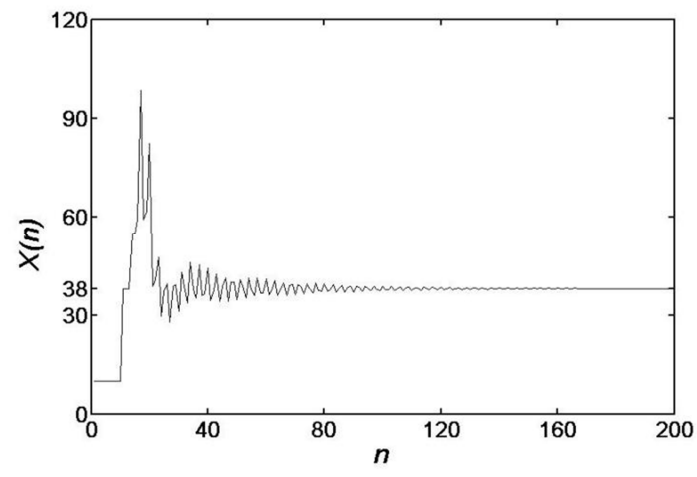

Figures 1. The solution of equation (4), when initial values $x_{0}=x_{-1}=\cdots=x_{-9}=10$.

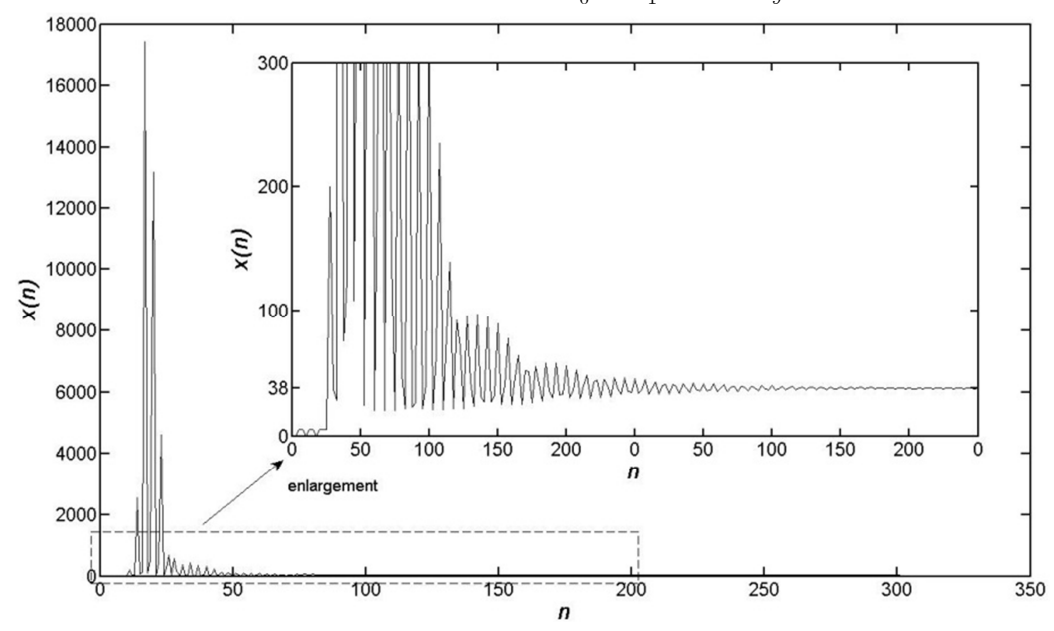

Figure 2. The solution of equation (4), when initial values $x_{-3}=x_{-6}=x_{-0}=0.05, \quad x_{0}=x_{-1}=x_{-2}=x_{-4}=x_{-5}=x_{-7}=x_{-8}=10$. 


\section{Conclusion}

In this paper, we investigate the characters of positive solution of the max-type difference equation (1).

First, we showed equation (1) has unique positive equilibrium $\bar{x}=p+A B$.

Then, we proved two useful lemmas. By citing lemmas we showed the main theorems in this paper, that is the equilibrium solution $\quad \bar{x}=p+A B \quad$ of equation (1) is globally asymptotically stable.

At last, we give an example of difference equation (1), draw the trajectory of the solution by giving two different initial values, thus intuitively reflect the global asymptotic stability.

\section{Acknowledgements}

Thanks for editors and reviewers' valuable comments and suggestions for improving this paper.

This research was supported by NNSF of China (11461007), Scientific and technological research project of Guangxi colleges and universities funded by Guangxi Department of Education (LX2014048, LX2014055), and Youth Foundation of Guangxi Normal University.

\section{References}

[1] El-Metwally H, "Global behavior of an economic model," Chaos Solitons \& Fractals, 33(3), 2007, pp. 994-1005.

[2] El-Metwally H, El-Afifi M M, "On the behavior of some extension forms of some population Models," Chaos Solitons \& Fractals, 36(1), 2008, pp. 104-114.

[3] Zhou L, Honghua H U, Liang C, et al, "Research on difference equation model in traffic flow calculation," Journal of Chang chun University of Science \& Technology, 2014, pp. 117-123.

[4] Huang C M, Wang W P, “Applications of difference equation in population forecasting model," Advanced Materials Research, 2014, pp. 1079-1080.

[5] Berenhaut K, Foley J, S. Stević S, "The global attractivity of the rational difference equation $y_{n}=1+y_{n-k} / y_{n-m}$," Proceedings of the American Mathematical Society, 135, 2007, pp. 1133-1140.

[6] Berenhaut K S, Stević S, "The behavior of the positive solutions of the difference equation $x_{n}=A+\left(x_{n-2} / x_{n-1}\right)^{p}$," J. Journal of Difference Equations and Applications, 12(9), 2006, pp. 909-918.
[7] Berg L, Stević S, "Periodicity of some classes of holomorphic difference equations," Journal of Difference Equations and Applications, 12(8), 2006, pp. 827-835.

[8] Iričanin B, Stević S, "Some systems of nonlinear difference equations of higher order with periodic solutions," Dynamics of Continuous Discrete and Impulsive Systems Series, 13A (3-4), 2006, pp. 499-507.

[9] Iričanin B, Stević S, "Eventually constant solutions of a rationa ldifference equation," Applied Mathematics and Computation, 215,2009 , pp. 854-856.

[10] Elabbasy E M, El-Metwally H A, Elsayed E M, "Global behavior of the solutions of some difference equations," Advances in Difference Equations, 28(2), 2011, pp. 683-689.

[11] Elsayed E M, Iričanin B, Stević S, "On the max-type equation $x_{n+1}=\max \left\{A_{n} / x_{n}, x_{n-1}\right\}$," Ars Combinatoria, 95, 2010, pp. 187-192.

[12] Stević S, "Global stability of a max-type difference equation," Applied Mathematics \& Computation, 216(1), 2010, pp. 354356.

[13] Sun T X, Xi H J, Han C H, "Dynamics of the max-type difference equation $x_{n+1}=\max \left\{1 / x_{n-m}, A_{n} / x_{n-r}\right\}$," Journal of Applied Mathematics and Computing, 2012 (1-2), 2012, pp. 173-180.

[14] Stević S, "On a symmetric system of max-type difference Equations," Applied Mathematics and Computation, 219(15), 2013, pp. 8407-8412.

[15] Stević S, "On some periodic systems of max-type difference equations," Applied Mathematics and Computation, 218, 2012, pp. 11483-11487.

[16] Amleh A M, Georgiou D A, Grove E A, Ladas G, "On the recursive sequence $x_{n+1}=\alpha+x_{n-1} / x_{n}$," Journal of Mathematital Analysis and applications, 233(2), 1999, pp. 790-798.

[17] Fan Y, Wang L, Li W, "Global behavior of a higher order nonlinear diference equation," Journal of Mathematital Analysis and applications, 299(1), 2004, pp. 113-126.

[18] Sun T X, He Q L, Wua X, Xi H J, "Global behavior of the max-type difference equation $x_{n+1}=\max \left\{1 / x_{n-m}, A_{n} / x_{n-r}\right\} \quad, \quad$ Applied Mathematics and Computation, 248, 2014, pp. 687-692.

[19] Liu W P, Stević S, "Global attractivity of a family of non-autonomous max-type difference equations," Applied Mathematics and Computation, 218(11), 2012, pp. 6297-9303.

[20] Grove E A, Ladas G, Periodicities in Nonlinear Difference Equations, Vol. 4, New York: Chapman\& Hall/CRC Press, 2005, pp. 2. 